Original Article

\title{
Relationship between metabolic syndrome and osteoarthritis: The Fasa Osteoarthritis Study
}

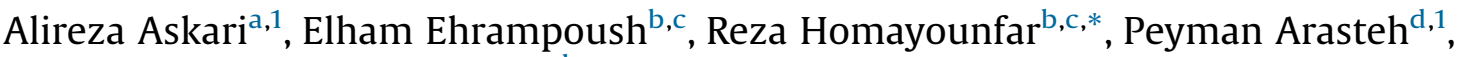 \\ Mohammad Mehdi Naghizadeh ${ }^{\mathrm{b}}$, Mohammadreza Yarahmadi ${ }^{\mathrm{e}}$, Niloofar Tarbiat ${ }^{\mathrm{e}}$, \\ Seyed Sajjad Eghbalif \\ a Department of Orthopedic surgery, Shiraz University of Medical Sciences, Shiraz, Iran \\ ${ }^{\mathrm{b}}$ Noncommunicable Diseases Research Center, Fasa University of Medical Sciences, Fasa, Iran \\ ${ }^{\mathrm{c}}$ Health Policy Research Center, Institute of Health, Shiraz University of Medical Sciences, Shiraz, Iran \\ d Trauma Research Center, Shahid Rajaee Trauma Hospital, Shiraz University of Medical Sciences, Shiraz, Iran \\ e Student Research Committee, Fasa University of Medical Sciences, Fasa, Iran \\ ${ }^{\mathrm{f}}$ Department of Pathology and Lab Medicine, Bushehr University of Medical Sciences, Bushehr, Iran
}

A R T I C L E I N F O

Article history:

Available online $\mathrm{xxx}$

Keywords:

Metabolic syndrome

Osteoarthritis

Body mass index

Waist circumference

Triglyceride

\begin{abstract}
A B S T R A C T
Background: An association between metabolic syndrome (MeS) and osteoarthritis (OA) has been reported in recent years; however, conflicting findings have been reported regarding this matter. Inhere we evaluated the relationship between different components of MeS and OA in a Fasa osteoarthritis registry (FOAS).

Methods: The registry includes all OA cases who referred to Fasa hospital (Iran) since 2013. Overall, 131 patients with OA with a Kellgren \& Lawrence (K\&L) score $>1$ and 261 controls were compared.

Results: Overall, $82.4 \%$ of individuals in the OA group and $40.8 \%$ of participants in the control group had MeS $(\mathrm{P}<0.001)$. Patients with $\mathrm{OA}$ had a 6.8 (95\% CI: 4.1-11.4) higher chance of acquiring MeS. After adjusting for sex, age, and BMI, odds' ratio (OR) for acquiring MeS in OA group increased to 10.9 (95\% CI: 5.5-21.8). Among MeS criteria's, high waist circumference (WC) has strongest correlation for acquiring OA (OR=27.535, 95\% CI: 6.003-126.306).

Conclusion: Our findings revealed that metabolic markers are strongly associated with OA and the addition of each component of the MeS, significantly increases the risk of developing OA, therefore control of metabolic factors and appropriate screening must be considered in health policy making and prevention programs.
\end{abstract}

(C) 2017 Diabetes India. Published by Elsevier Ltd. All rights reserved.

\section{Introduction}

Osteoarthritis (OA) is one of the most disabling diseases afflicting the elderly population. It has been estimated that, approximately $25 \%$ of people over the age of 55 years old, experience permanent pain and roughly $10 \%$, experience debilitating pain in their knees [1]. Based on disability adjusted life years (DALY) an annual estimated 2,118,000 years of human lives are lost due to the disease [2]. OA mostly targets middle aged people (4565 years) [3] and patients usually go through a rapid course of

\footnotetext{
* Corresponding author at: Noncommunicable Diseases Research Center, Fasa University of Medical Sciences, Fasa, Iran.

E-mail addresses: r_homayounfar@yahoo.com, homayounfar@sbmu.ac.ir (R. Homayounfar).

1 These two authors have equal contribution to this work.
}

disease progression [4]. Sex, weight, injuries to the joint, genetic and metabolic disorders are among factors believed to play a role in OA pathogenesis $[5,6]$, however etiology of the disease is not fully recognized.

The link between metabolic disorders and OA has been a subject of interest in recent years to the extent that the idea of metabolic OA has been proposed [7]. Most studies evaluating the connection between metabolic markers and OA, have focused their attention on $\mathrm{OA}$ of the hand, since it is least affected by the imposed weight load on joints. However, distinct characteristics of different joints affect their susceptibility to diseases [8].

Metabolic syndrome (MeS) is characterized by insulin resistance, dyslipidemia, hypertension and abdominal obesity which can lead to diseases such as type II diabetes and cardiovascular diseases [9-11]. Even though the definition of MeS is highly variable for different population [7,12], studies show that about $59 \%$ of OA patients have MeS $[4,13]$. 
To date, several studies have reported conflicting findings regarding the relationship between OA and MeS. These studies have mostly failed to find a significant connection between MeS and the risk of $\mathrm{OA}$, after adjusting for variables such as weight or body mass index (BMI). Inhere we evaluated the relationship between different components of MeS and OA in a cohort registry entitled the Fasa Osteoarthritis Study (FOAS).

\section{Patients and methods}

\subsection{Study protocol}

This study is part of an OA registry affiliated to Fasa University of Medical Sciences termed the Fasa osteoarthritis study (FOAS). The registry includes all OA cases who referred to Fasa hospital (Iran) since 2013. Currently the database includes 131 registered patients with OA.

Diagnosis of OA was based on having a Kellgren \& Lawrence (K\&L) score $>1$ [14]. We considered a control group of 262 individuals from those referring to the orthopedic clinic of the hospital without complaints of pain and/or stiffness in their knee or hip. The inclusion criteria for the control group was lack of radiographic complication caused by OA in the knee and hip (K\&L score $=0$ ).

The case and control groups were matched according to gender. Blood samples were taken from all patients in one day at the central laboratory of the University.

\subsection{Measurements}

For the anthropometric measurements participants stood bare footed with light clothing. Waist circumference (WC) was considered at midpoint of the inferior border of the lowest ribs to the anterior superior iliac spine, after the participant had a normal expiration, using an inelastic tape.

For the anthropometry calculations weight was measured to the nearest $0.1 \mathrm{~kg}$ using a digital scale (Seca 767, Japan) and height was also measured to the nearest $0.1 \mathrm{~cm}$ using a stadiometer (Seca 767, Japan). Anthropometric index included BMI, which was considered weight divided by the square of height $\left(\mathrm{kg} / \mathrm{m}^{2}\right)$.

Other measurements included blood pressure (BP), which was done using a sphygmomanometer (Erka Perfect Aneroid, Germany). For evaluating BP, participants first rested for ten minutes, after which with an interval of five minutes, three consecutive BP's were measured from participants' right arm and a mean was calculated and considered the final BP.

Blood work included triglyceride (TG), cholesterol (Cho), low density lipoprotein cholesterol (LDL-c), high density lipoprotein cholesterol (HDL-c), and fasting blood sugar (FBS), which were all obtained from venous blood samples.

Laboratory testing of FBS was done using a glucose oxidize test (intra- and inter-assay coefficients of variation less than 2.1 and 2.6, respectively). For lipid profile an enzymatic approach (Parsazmoon Inc., Tehran, Iran), was used. We used the Friedewald formula to calculate LDL-c levels [15].

The National Cholesterol Education Program's Adult Treatment Panel (ATP) III revised guidelines were used for the diagnosis of MeS. Those participants who had three or more of the following criteria were considered having MeS: systolic BP $>130 \mathrm{mmHg}$ and/ or diastolic BP $>85 \mathrm{mmHg}$, TG $\geq 150 \mathrm{mg} / \mathrm{dl}$, HDL-c $<40 \mathrm{mg} / \mathrm{dl}$ for male and $<50 \mathrm{mg} / \mathrm{dl}$ for females, FBS $>100$ and $\mathrm{WC}>88 \mathrm{~cm}$ for women or WC $>102 \mathrm{~cm}$ for men [16].
For evaluating pain, the Western Ontario and McMaster universities arthritis index score (WOMAC) was also measured [17].

\subsection{Ethical consideration}

The study protocol followed the principals of the "Declaration of Helsinki". The Institutional Review Board (IRB) of Fasa University of Medical Sciences approved the study. Written and informed consents were obtained from all the participating in the study.

\subsection{Statistical analysis}

Data was analyzed using SPSS ${ }^{\circledR}$ for windows ${ }^{\circledR}$, version 22 (SPSS Inc., Chicago, IL, USA).

Comparison of means of normally distributed variables between the $\mathrm{OA}$ and the control group was done using the independent $t$-test and the chi-square test for quantitative and qualitative variables, respectively. The multiple logistic regression model was used to evaluate the effect of MeS on OA. In our multivariate analysis, we added each of the components of the MeS in a separate manner to evaluate the independent effects of each component of MeS on OA, furthermore the model was adjusted for sex, age and BMI for each of the analysis.

The linear regression model was used to determine WOMAC score correlates. We also reported the Wald statistics to evaluate the power of association of each variable with OA.

All data are presented as means and standard deviations (SD) or percentage and frequency, where appropriate. Our statistical inference was based on a 95\% confidence interval (CI) and a significance threshold of $5 \%$.

\section{Results}

Baseline characteristics of patients are displayed in Table 1.

Hypertension, abnormal FBS, TG, and HDL (in the context of the definition of MeS) were significantly higher among (96.2\%, 20.6\%, $75.6 \%$, and $26.7 \%$, respectively) individuals with OA in comparison to participants in the control group $(81.3 \%, 12.6 \%, 37 \%$, and $18.7 \%$, respectively) ( $\mathrm{p}<0.05)$

Overall, $82.4 \%$ of individuals in the OA group and $40.8 \%$ of participants in the control group had MeS $(\mathrm{P}<0.001)$ (Table 2$)$.

In our univariate analysis patients with OA showed a $6.8(95 \%$ $\mathrm{CI}$ : 4.1-11.4) higher chance of acquiring $\mathrm{MeS}$ in comparison to those in the control group. After adjusting for sex, age, and BMI, odds' ratio (OR) for acquiring $\mathrm{MeS}$ in patients with $\mathrm{OA}$ in comparison to participants in the control group increased to 10.9 (95\% CI: 5.5-21.8).

Table 1

Patients' baseline characteristics.

\begin{tabular}{llll}
\hline Variables & Control $(\mathrm{n}=262)$ & Osteoarthritis $(\mathrm{n}=131)$ & p-value \\
\hline Age $-\mathrm{yrs}$ & $55.49 \pm 9.12$ & $52.86 \pm 8.77$ & 0.007 \\
Waist $-\mathrm{cm}$ & $86.89 \pm 4.76$ & $91.57 \pm 4.87$ & $<0.001$ \\
$\mathrm{BMI}-\mathrm{kg} / \mathrm{m}^{2}$ & $25.97 \pm 2.38$ & $28.24 \pm 2.19$ & $<0.001$ \\
$\mathrm{FBS}-\mathrm{mg} / \mathrm{dl}$ & $82.91 \pm 21.78$ & $98.34 \pm 18.45$ & $<0.001$ \\
$\mathrm{TG}-\mathrm{mg} / \mathrm{dl}$ & $137.10 \pm 38.17$ & $178.37 \pm 42.55$ & $<0.001$ \\
$\mathrm{Chol}-\mathrm{mg} / \mathrm{dl}$ & $151.13 \pm 38.90$ & $196.91 \pm 43.07$ & $<0.001$ \\
$\mathrm{HDL}-\mathrm{mg} / \mathrm{dl}$ & $58.20 \pm 11.22$ & $54.52 \pm 11.02$ & 0.002 \\
LDL $-\mathrm{mg} / \mathrm{dl}$ & $84.99 \pm 24.02$ & $105.17 \pm 22.83$ & $<0.001$ \\
Systolic BP $-\mathrm{mmHg}$ & $13.34 \pm 1.21$ & $14.42 \pm 1.40$ & $<0.001$ \\
Diastolic BP $-\mathrm{mmHg}$ & $8.71 \pm 1.03$ & $9.30 \pm 1.05$ & $<0.001$ \\
WOMAC score & $5.50 \pm 2.80$ & $15.11 \pm 3.25$ & $<0.001$ \\
K\&L & 0 & $2.93 \pm 0.59$ & $<0.001$ \\
\hline
\end{tabular}

FBS: fasting blood sugar; TG: triglyceride; Chol: cholesterol; HDL: high density lipoprotein; LDL: low density lipoprotein; WOMAC: Western Ontario and McMaster universities arthritis index; K\&L: Kellgren \& Lawrence. 
Table 2

Comparison of osteoarthritis and control groups.

\begin{tabular}{|c|c|c|c|}
\hline Variables & Control $(n=262)$ & Osteoarthritis $(n=131)$ & p-value \\
\hline \multicolumn{4}{|l|}{ Sex } \\
\hline Male & $48(18.3)$ & $24(18.3)$ & \multirow[t]{2}{*}{1.000} \\
\hline Female & $214(81.7)$ & $107(81.7)$ & \\
\hline \multicolumn{4}{|l|}{$\mathrm{HDL}-\mathrm{mg} / \mathrm{dl}$} \\
\hline Normal & $213(81.3)$ & $96(73.3)$ & \multirow[t]{2}{*}{0.068} \\
\hline Abnormal & $49(18.7)$ & $35(26.7)$ & \\
\hline \multicolumn{4}{|l|}{$\mathrm{WC}-\mathrm{cm}$} \\
\hline Normal & $46(17.6)$ & $5(3.8)$ & \multirow[t]{2}{*}{$<0.001$} \\
\hline Abnormal & $216(82.4)$ & $126(96.2)$ & \\
\hline \multicolumn{4}{|c|}{$\mathrm{HTN}-\mathrm{mmHg}$} \\
\hline Normal & $49(18.7)$ & $5(3.8)$ & \multirow[t]{2}{*}{$<0.001$} \\
\hline Abnormal & $213(81.3)$ & $126(96.2)$ & \\
\hline \multicolumn{4}{|l|}{ FBS $-\mathrm{mg} / \mathrm{dl}$} \\
\hline Normal & $229(87.4)$ & $104(79.4)$ & \multirow[t]{2}{*}{0.037} \\
\hline Abnormal & $33(12.6)$ & $27(20.6)$ & \\
\hline \multicolumn{4}{|l|}{$\mathrm{TG}-\mathrm{mg} / \mathrm{dl}$} \\
\hline Normal & $165(63.0)$ & $32(24.4)$ & \multirow[t]{2}{*}{$<0.001$} \\
\hline Abnormal & $97(37.0)$ & $99(75.6)$ & \\
\hline \multicolumn{4}{|l|}{ MeS } \\
\hline No & $155(59.2)$ & $23(17.6)$ & \multirow[t]{2}{*}{$<0.001$} \\
\hline Yes & $107(40.8)$ & $108(82.4)$ & \\
\hline \multicolumn{4}{|c|}{ MeS number of components } \\
\hline 0 & $4(1.5)$ & $0(0.0)$ & \multirow{6}{*}{$<0.001$} \\
\hline 1 & $44(16.8)$ & $1(0.8)$ & \\
\hline 2 & $107(40.8)$ & $22(16.8)$ & \\
\hline 3 & $79(30.2)$ & $67(51.1)$ & \\
\hline 4 & $27(10.3)$ & $38(29.0)$ & \\
\hline 5 & $1(0.4)$ & $3(2.3)$ & \\
\hline
\end{tabular}

WC: waist circumference; HTN: hypertension; FBS: fasting blood sugar; TG: triglyceride; MeS: metabolic syndrome.

By separating the components of MeS, we found that with adding each of the components of MeS, OR for acquiring OA increases subsequently.

When evaluating the five component of MeS separately, high TG, high WC, low HDL, and abnormal FBS (in the context of the definition of MeS) were correlated with MeS $(\mathrm{p}<0.05)$. Among these factors, high TG had the strongest correlation with OA (Wald statistics $=31.8, \mathrm{OR}=6.02,95 \% \mathrm{CI}: 3.228-11.255)$, furthermore high WC was another strong correlate for acquiring OA (Wald statistics $=18.2, \mathrm{OR}=27.535,95 \% \mathrm{CI}: 6.003-126.306)$. Hypertension did not show a statistically significant correlation with OA (Wald statistic $=3.75$, OR = 3.32, 95\% CI: 0.986-11.21) (Table 3).

Table 3

Multivariate analysis for evaluating the relationship between osteoarthritis and metabolic syndrome components in adjusted and non-adjusted models.

\begin{tabular}{|c|c|c|c|c|}
\hline Variables & OR & 95\% Confidence interval & Wald statistics & p-value \\
\hline No MeS (0 to 2 factors) & & & 50.495 & $<0.001$ \\
\hline Unadjusted model of MeS & 6.802 & $4.072-11.363$ & 53.634 & $<0.001$ \\
\hline Adjusted model of MeS & 10.945 & $5.496-21.796$ & 46.349 & $<0.001$ \\
\hline Female sex & 1.780 & $0.735-4.311$ & 1.630 & 0.202 \\
\hline Age & 0.868 & $0.830-0.907$ & 39.232 & $<0.001$ \\
\hline BMI & 1.972 & $1.686-2.308$ & 71.858 & $<0.001$ \\
\hline MeS with 3 components & 8.444 & $4.112-17.340$ & 33.769 & $<0.001$ \\
\hline MeS with 4 components & 20.189 & $8.277-49.244$ & 43.636 & $<0.001$ \\
\hline MeS with 5 components & 31.465 & $2.708-365.596$ & 7.596 & 0.006 \\
\hline Female sex & 1.772 & $0.727-4.320$ & 1.581 & 0.209 \\
\hline Age & 0.864 & $0.826-0.904$ & 40.477 & $<0.001$ \\
\hline BMI & 2.003 & $1.705-2.354$ & 71.510 & $<0.001$ \\
\hline \multicolumn{5}{|l|}{ MeS components } \\
\hline $\mathrm{TG}^{\mathrm{a}}$ & 6.028 & $3.228-11.255$ & 31.798 & $<0.001$ \\
\hline Waist $^{\mathrm{b}}$ & 27.535 & $6.003-126.306$ & 18.199 & $<0.001$ \\
\hline $\mathrm{HDL}^{\mathrm{c}}$ & 2.613 & $1.292-5.286$ & 7.138 & 0.008 \\
\hline FBS $^{\mathrm{d}}$ & 2.393 & $1.086-5.273$ & 4.689 & 0.030 \\
\hline $\mathrm{HTN}^{\mathrm{e}}$ & 3.325 & $0.986-11.218$ & 3.750 & 0.053 \\
\hline Female sex ${ }^{\mathrm{f}}$ & 1.052 & $0.356-3.111$ & 0.008 & 0.927 \\
\hline Age & 0.859 & $0.820-0.900$ & 40.436 & $<0.001$ \\
\hline BMI & 2.016 & $1.701-2.390$ & 65.510 & $<0.001$ \\
\hline
\end{tabular}

MeS: metabolic syndrome; BMI: body mass index; TG: triglyceride; HDL: high density lipoprotein; FBD: fasting blood sugar; HTN: hypertension.

a TG was considered as $>150 \mathrm{mg} / \mathrm{dL}$.

b Waist was considered as $>94$ for males and $>80$ for female.

c HDL was considered as $<40$ for males and $<50$ for females.

d FBS was considered as $>110 \mathrm{mg} / \mathrm{dl}$.

e Hypertension was considered as $>130 \mathrm{mmHg}$ for systolic blood pressure or $>85 \mathrm{mmHg}$ for diastolic blood pressure.

f Sex, age and BMI were adjusted for in all the analysis. 
Table 4

Linear regression analysis for evaluating the relationship between WOMAC score and different variables.

\begin{tabular}{lllll}
\hline Variables & Beta & 95\% confidence interval & P-value & $\mathrm{R}^{2}$ of model \\
\hline Constant & 6.57 & $3.188-9.953$ & $<0.001$ & 0.704 \\
MeS & -0.371 & -1.063 to 0.321 & 0.292 & \\
OA & 9.894 & $9.065-10.722$ & $<0.001$ & \\
Sex & -0.197 & -1.033 to 0.638 & 0.643 & \\
Age & 0.003 & -0.038 to 0.045 & 0.872 & \\
BMI & -0.039 & -0.192 to 0.114 & 0.620 & \\
\hline
\end{tabular}

MeS: metabolic syndrome; OA: osteoarthritis; BMI: body mass index.

Linear regression analysis showed that in patients with $\mathrm{OA}, \mathrm{MeS}$ was not correlated with WOMAC score after adjusting for sex, age and BMI $(\mathrm{P}=0.292)$ (Table 4).

\section{Discussion}

In this study, we investigated the relationship between MeS and OA. Our results showed that the prevalence of MeS in OA patients was higher than those in the control group. When we adjusted for age, sex and BMI, the correlation between MeS and OA increased (from 6.8 to 10.94). Our study showed that by an increase in each of the components of MeS, the risk of acquiring OA rises significantly (patients who have all five components of MeS and those who have 4 components of the MeS are 31 times and 20 times more likely to develop OA than patients who do not have MeS). To the best of the authors' knowledge, this is the only study that has linked MeS by each of its components to the risk of developing OA.

The work by Puenpatom et al. [4] can be considered among the very first studies that pointed to the relationship between MeS and OA. Utilizing the results of a larger study termed the "NHANES III" study, they found that $59 \%$ of the population with OA had MeS, compared to only $23 \%$ of the population who did not have OA.

According to their study, all complications associated with MeS including: high BP (75\% vs. $38 \%$ ), abdominal obesity (63\% vs. $38 \%$ ), hyperglycemia ( $30 \%$ vs. $13 \%$ ), high triglycerides ( $47 \%$ vs. $32 \%$ ), and high LDL (44\% vs. 38\%), had higher frequency among patients with $\mathrm{OA}$ in comparison to an apparently healthy group. They also showed that the relationship between MeS and OA is stronger at younger ages and weakens as patients grow older.

Marksabcdef et al. [18] studied 1000 patients with hip OA to evaluate accompanying diseases. They found that more than $55 \%$ of patients had at least one comorbidity such as hypertension or cardiovascular disease.

In a cross-sectional study in 2013, Morovic-Vergles et al. [19], found that among 352 patients with OA, 60\% had hypertension after adjusting for age and BMI.

Schett et al. [20], using data from the "Bruneck Study", found that arthroplasty rate was 17.7 (range: 9.4-30.2) in patients with diabetes type II per 1000 patient-years. They concluded that diabetes is an independent risk factor for arthroplasty, with a hazard ratio of 2.1 (range: $1.1-3.8$ ), after adjusting for age, sex, BMI and prior joint replacements.

A study carried out on 100 diabetic and 102 non-diabetic individuals by Nieves-Plaza et al. [21] found that the incidence of OA in diabetics was $49 \%$, while the incidence in non-diabetics was 26.5\% $(\mathrm{p}<0.01)$. Multivariate analysis showed that diabetic patients have an OR of 2.18 for acquiring OA (95\% CI: $1.12-4.24)$ compared to non-diabetics.

Unfortunately, the majority of studies that investigated the relationship between $\mathrm{MeS}$ and $\mathrm{OA}$, although successful at finding a relationship in their early stages of analysis, were unable to find a significant link between the two, after adjusting for BMI. Based on the "Korean National Health and Nutrition Examination Survey",
Han et al. [22] initially found a positive and significant relationship between MeS and OA in women $(\mathrm{OR}=1.798,95 \% \mathrm{CI}$ : 1.392-2.322). However, after adjusting for confounders such as age, alcohol consumption, and smoking status, they found that only WC was positively associated with increased risk of knee $\mathrm{OA}(\mathrm{OR}=1.117,95 \%$ CI: 0.805-1.550). The study found no relationship between MeS and $\mathrm{OA}$ in men.

In a cohort study by Engstrom et al. [23] they found that MeS was associated with increased incidence of OA of the knee. The relationship continued to exist even after adjusting for sex, age, smoking, and physical activity. However, the positive correlation was non-exiting after adjusting for BMI. One of the interesting findings of this study was that they documented a different response to MeS based on the involved joints (hip or knee).

The relationship between diabetes and OA is also a subject of controversy. Early studies did not find any association between these two diseases, in addition the results of the "First National Health and Nutrition Examination Survey" also found a lack of association between the two conditions [24]. This finding was also confirmed by other studies [25,26]. In general, studies found that other than BMI and obesity, no other metabolic variable relates to OA.

In a cohort study with a population of 6197, Garessus et al. [27] calculated an adjusted OR of 1.18 per one unit increase in blood sugar (serum glucose). They found no significant relationship between metabolic markers of blood sugar including glucose levels, insulin, HbA1C or homeostatic model assessment-IR, and the occurrence of OA. It seems that since the study examined patients with normal BMI (average BMI of the population was 26 and ranged from 22 to 30), they were not been able to show a strong and significant correlation between the two variables.

In our study, BP was not significantly associated with OA $(\mathrm{OR}=3.325,95 \% \mathrm{CI}: 0.986-11.218)$. It seems that even after adjusting for variables such as sex, age, and BMI, BP would still have an insignificant association with $\mathrm{OA}$ compared to other components of the MeS.

Among MeS components, TG and WC had the strongest correlation with OA. The most interesting finding in our study, which we showed for the first time, was that perhaps TG (as part of the MeS) may play a crucial role in the pathogenesis of OA and has the strongest association with $\mathrm{OA}$, even after adjusting for other variables. Therefore, we recommend further studies evaluate the role of TG in the pathogenesis of OA.

We also found that WC, independent from BMI, which has been mentioned as a major risk factor by most studies, is strongly correlated with OA. Aside to the increased mechanical load imposed on the joints due to high BMI, abdominal obesity seems to independently take part in $\mathrm{OA}$ pathogenesis due its unique role with systematic inflammatory mediators and adipokines in the body [28].

In line with existing studies [20,29], our study showed that metabolic markers can add to the risk of OA. The addition of each component of the MeS, significantly increases the risk of developing OA. However, several research studies revealed no association between the prevalence of OA and metabolic markers. Numerous factors may have clouded the relationship between OA and MeS, including discrepancies associated with study population, race, sample size, life style, and diet. Moreover, different diagnostic methods and procedures to evaluate OA like radiological findings, self-assessment or symptom revision, may have also played a role in this matter. Even the criteria for diagnosis of MeS were different among studies [7,30].

The relationship between the two conditions has only been reported significant, when studies considered MeS with all of its criteria [29,31,32]. 
The relationship between obesity and OA, due to the increased load on the joints, has long been recognized [13]. However, we found that MeS components each play an independent role in $\mathrm{OA}$ pathogenesis.

We also found that risk of acquiring MeS did not have a notable impact on the level of pain perceived by patients with OA.

In this study, we employed the K\&L method to diagnose and differentiate between case and control groups, so the participants who entered the control group had no OA related complication. Perhaps the meaningful difference between the case and control groups found in our study was due to this matter. Nonetheless, our study had some limitations. The cross-sectional nature of the study did not allow us to examine causality or eliminate reverse causation relationships. Despite great efforts to reduce confounding factors, due to the observational nature of the study these factors still exist. We only examined the knee joint and as studies have indicated, there may be difference in different joints regarding their association with metabolic factors. However, we did adjust for BMI to eliminate this difference. Although we did not separate ethnic groups, our study included different ethnic groups including Turk, Farsi and Arab, which is among the strong point of our study. Lifestyle differences and different socio-economic status between these ethnic groups are factors which cannot be resolved.

In conclusion, our findings revealed that metabolic markers are strongly associated with OA, therefore control of metabolic factors and appropriate screening must be considered in health policy making and prevention programs, especially in Iran where the rates of MeS are high compared to other countries [12]. More specifically, special attention should be given to abdominal obesity (WC) and hyperlipidemia. Excess fat in the abdominal area can have an impact on $\mathrm{OA}$, which is independent from the general obesity measured using BMI.

\section{Conflict of interest}

There is no conflict of interest to be declared regarding the manuscript.

\section{Author contributions}

Conceptualization: RH EE.

Methodology: RH EE.

Software: MMN.

Validation: RH MMN.

Formal analysis: MMN.

Investigation: MY NT SSE.

Resources: AA RH EB.

Data curation: RH MMN.

Writing (original draft preparation): RH PA.

Writing (review and editing): PA RH SSE.

Visualization: AA.

Supervision: RH.

Project administration: $\mathrm{RH}$.

Funding acquisition: AA RH.

\section{Classification}

Metabolic disease.

\section{Implication for health policy makers/practice/research/medical education}

Determining the relationship between metabolic disease and osteoarthritis, which causes the loss of a yearly 2118000 daily adjusted life years, is of paramount importance for health related policy making and preventive programs. These especially influence countries that are adapting to a western life style and have increasing rates of metabolic disease.

\section{Acknowledgements}

This work is part of a large registry of osteoarthritis patients in the south east of Fars province, Iran which is funded by Fasa University of Medical Sciences. Clinical research development unit supports the project by the grant number 93128. There is no independent financial support for the current work. Other study results of the registry are in press. We would also like to thank all the individuals who patiently participated in our study.

\section{References}

[1] Peat G, McCarney R, Croft P. Knee pain and osteoarthritis in older adults: a review of community burden and current use of primary health care. Ann Rheum Dis 2001;60(2):91-7.

[2] Lopez AD, Mathers CD, Ezzati M, Jamison DT, Murray CJ. Global and regional burden of disease and risk factors, 2001: systematic analysis of population health data. Lancet 2006;367(9524):1747-57.

[3] Bijlsma JW, Berenbaum F, Lafeber FP. Osteoarthritis: an update with relevance for clinical practice. Lancet 2011;377(9783):2115-26.

[4] Puenpatom RA, Victor TW. Increased prevalence of metabolic syndrome in individuals with osteoarthritis. Postgrad Med 2009121(6).

[5] Goldring MB, Goldring SR. Osteoarthritis. J Cell Physiol 2007;213(3):626-34.

[6] Shoara R, Hashempur MH, Ashraf A, Salehi A, Dehshahri S, Habibagahi Z. Efficacy and safety of topical Matricaria chamomilla L. (chamomile) oil for knee osteoarthritis: a randomized controlled clinical trial. Complement Ther Clin Pract 2015;21(3):181-7.

[7] Zhuo Q, Yang W, Chen J, Wang Y. Metabolic syndrome meets osteoarthritis. Nat Rev Rheumatol 2012;8(12):729-37.

[8] Yusuf E. Metabolic factors in osteoarthritis: obese people do not walk on their hands. Arthritis Res Ther 2012;14(4):123.

[9] Alberti K, Eckel RH, Grundy SM, Zimmet PZ, Cleeman JI, Donato KA, et al. Harmonizing the metabolic syndrome a joint interim statement of the international diabetes federation task force on epidemiology and prevention; national heart, lung, and blood institute; American heart association; world heart federation; international atherosclerosis society; and international association for the study of obesity. Circulation 2009;120(16):1640-5.

[10] Askari A, Naghizadeh MM, Homayounfar R, Shahi A, Afsarian MH, Paknahad A, et al. Increased serum levels of IL-17A and IL-23 are associated with decreased vitamin D3 and increased pain in osteoarthritis. PLoS One 2016;11(11): e0164757.

[11] Zand H, Homayounfar R, Cheraghpour M, Jeddi-Tehrani M, Ghorbani A, Pourvali K, et al. Obesity-induced p53 activation in insulin-dependent and independent tissues is inhibited by beta-adrenergic agonist in diet-induced obese rats. Life Sci 2016;147:103-9.

[12] Babai MA, Arasteh P, Hadibarhaghtalab M, Naghizadeh MM, Salehi A, Askari A et al. Defining a BMI cut-off point for the iranian population: the shiraz heart study. PLoS One 2016;11(8):e0160639.

[13] Askari A, Ehrampoush E, Homayounfar R, Bahramali E, Farjam M. Serum insulin in pathogenesis and treatment of osteoarthritis. Med Hypotheses 2017;99:45-6.

[14] Schiphof D, Boers M, Bierma-Zeinstra SM. Differences in descriptions of Kellgren and Lawrence grades of knee osteoarthritis. Ann Rheum Dis 2008;67 (7):1034-6.

[15] Zeng Q, He Y, Dong S, Zhao X, Chen Z, Song Z, et al. Optimal cut-off values of BMI, waist circumference and waist:height ratio for defining obesity in Chinese adults. Br J Nutr 2014;112(10):1735-44.

[16] Grundy SM, Cleeman JI, Daniels SR, Donato KA, Eckel RH, Franklin BA, et al. Diagnosis and management of the metabolic syndrome: an American Heart Association/National Heart, Lung, and Blood Institute Scientific Statement. Circulation 2005;112(17):2735-52.

[17] Goggins J, Baker K, Felson D. What WOMAC pain score should make a patient eligible for a trial in knee osteoarthritis? J Rheumatol. 2005;32(3):540-2.

[18] Marksabcdef R, Allegranteeg JP. Comorbid disease profiles of adults with endstage hip osteoarthritis. Med Sci Monit 2002;8(4):CR305-9.

[19] Morović-Vergles J, Šalamon L, Marasović-Krstulović D, Kehler T, Šakić D, Badovinac $\mathrm{O}$, et al. Is the prevalence of arterial hypertension in rheumatoid arthritis and osteoarthritis associated with disease? Rheumatol Int 2013;33 (5):1185-92.

[20] Schett G, Kleyer A, Perricone C, Sahinbegovic E, Iagnocco A, Zwerina J, et al. Diabetes is an independent predictor for severe osteoarthritis results from a longitudinal cohort study. Diabetes Care 2013;36(2):403-9.

[21] Nieves-Plaza M, Castro-Santana LE, Font YM, Mayor AM, Vilá LM. Association of hand or knee osteoarthritis with diabetes mellitus in a population of Hispanics from Puerto Rico. J Clin Rheumatol 201319(1). 
[22] Han CD, Yang IH, Lee WS, Park YJ, Park KK. Correlation between metabolic syndrome and knee osteoarthritis: data from the Korean National Health and Nutrition Examination Survey (KNHANES). BMC Public Health 2013;13(1):1.

[23] Engström G, Gerhardsson de Verdier M, Rollof J, Nilsson P, Lohmander L. Creactive protein, metabolic syndrome and incidence of severe hip and knee osteoarthritis. A population-based cohort study. Osteoarthritis Cartilage 2009;17(2):168-73.

[24] Anderson JJ, Felson DT. Factors associated with osteoarthritis of the knee in the first national Health and Nutrition Examination Survey (HANES I) evidence for an association with overweight, race, and physical demands of work. Am J Epidemiol 1988:128(1):179-89.

[25] Frey M, Barrett-Connor E, Sledge P, Schneider D, Weisman M. The effect of noninsulin dependent diabetes mellitus on the prevalence of clinical osteoarthritis. A population based study. J Rheumatol 1996;23(4):716-22.

[26] Martin K, Lethbridge-Cejku M, Muller D, Elahi D, Andres R, Tobin J, et al. Metabolic correlates of obesity and radiographic features of knee osteoarthritis: data from the Baltimore Longitudinal Study of Aging. J Rheumatol 1997;24(4):702-7.
[27] Garessus E, de Mutsert R, Visser A, Rosendaal F, Kloppenburg M. No association between impaired glucose metabolism and osteoarthritis. Osteoarthritis Cartilage 2016;24(9):1541-7.

[28] Lubberts E. The IL-23-IL-17 axis in inflammatory arthritis. Nat Rev Rheumatol 2015.

[29] Dahaghin S, Bierma-Zeinstra S, Koes B, Hazes J, Pols H. Do metabolic factors add to the effect of overweight on hand osteoarthritis? The Rotterdam Study. Ann Rheum Dis 2007;66(7):916-20.

[30] Ehrampoush E, Arasteh P, Homayounfar R, Cheraghpour M, Alipour M, Naghizadeh MM, et al. New anthropometric indices or old ones: which is the better predictor of body fat? Diabetes Metab Syndr: Clin Res Rev 2016.

[31] Hussain SM, Wang Y, Cicuttini FM, Simpson JA, Giles GG, Graves S, et al Incidence of total knee and hip replacement for osteoarthritis in relation to the metabolic syndrome and its components: a prospective cohort study Elsevier Semin Arthritis Rheum 2014.

[32] Yoshimura N, Muraki S, Oka H, Tanaka S, Kawaguchi H, Nakamura K, et al Accumulation of metabolic risk factors such as overweight, hypertension, dyslipidaemia, and impaired glucose tolerance raises the risk of occurrence and progression of knee osteoarthritis: a 3-year follow-up of the ROAD study. Osteoarthritis Cartilage 2012;20(11):1217-26. 